\title{
Proceedings of the 15th European Powder Diffraction Conference
}

This special issue of Powder Diffraction offers a selection of contributions presented at the 15th European Powder Diffraction Conference (EPDIC15) held in Bari, a coastal town in southern Italy, from June 12 to $15,2016$.

Starting in Munich in 1991, the EPDIC conferences have rapidly become reference point for all researchers involved in studies of polycrystalline materials by diffraction methods. The EPDIC conferences are now regarded as the ideal venue for the presentation and diffusion of new developments in powder diffraction instrumentation, analysis, and applications. The next conference, EPDIC16, will take place in Edinburgh, Scotland, on July 1-4, 2018. Details of the EPDIC organization can be found at http://epdic.ing.unitn.it/index.html.

The year 2016 has been a landmark year for the history of powder diffraction: almost a century has passed since the pioneering works and experiments of Debye, Scherrer, and Hull; 50 years have passed since the first publication of the Rietveld method. Because of this, the EPDIC15 conference opened with a plenary lecture, brilliantly given by Professor William David celebrating the 50 years of the Rietveld method.

Two invited Award Talks were delivered: the first ("Phasing from Powder Data: EXPO in a dynamic scientific world") during the opening ceremony by Carmelo Giacovazzo, winner of the EPDIC Distinguished Powder Diffractionist Award, and the second ("Total Scattering and Nanostructure Analysis for Materials Chemistry") during the closing ceremony by Kirsten Marie Ørnsbjerg Jensen, winner of the EPDIC Young Scientist Award.

In agreement with the traditional character of the EPDIC conferences, EPDIC15 provided an attractive scientific program covering a wide range of hot topics: total scattering and disorder; crystallography, and structural studies; biological, pharmaceutical, and biomedical materials; materials for sustainable development; combined methods for structure investigation and imaging; progress in instrumentation; diffraction line profile analysis, stress, strain, and texture; in situ/operando studies; nanomaterials, surfaces, and interfaces; neutron structural and magnetic scattering; and highpressure diffraction.

Plenary talks focused on the following topics: X-ray crystallography at the energy frontier (by K. W. Chapman); Magnetic structures (by A. S. Wills); Diffraction under imaging conditions (by T. U. Schulli); Structure, dynamics, and phase transitions of hybrid perovskite photovoltaics (by P. S. Whitfield); In situ diffraction under complex loading conditions (by S. Van Petegem); and Minerals and mineral-inspired materials for sustainable applications (by G. Cruciani).

The remainder of the program was organized in 13 microsymposia, each providing keynote talks and contributed oral presentations as well as two poster sessions, and the Software Fayre dedicated to the memory of Lachlan Cranswick.

About 360 persons attended EPDIC15 from 37 countries, including 19 countries from outside Europe.

The EPDIC15 conference has been a lively occasion for the powder diffraction community, always interested to gather information of recent progress in diffraction methods and applications, to share skills and results, and spread knowledge in Europe and, more generally, in the world.

The guest editors of this special issue of Powder Diffraction:

\author{
Michela Brunelli \\ SNBL/ESRF, Grenoble, France \\ Paolo Scardi \\ University of Trento, Trento, Italy \\ Angela Altomare
}

Institute of Crystallography-CNR, Bari, Italy 